\title{
A Modified Branched Graft Inversion Technique Employing a Syringe Inserter in Ascending Aortic Replacement for Type A Aortic Dissection
}

\author{
Masashi Kawabori*, Takashi Murakami, Kuntae Ahn, Yuji Kato, Mitsuhisa Kotani, \\ Masaaki Toyama \\ Department of Cardiovascular Surgery, Kameda General Hospital, Chiba, Japan \\ Email: ${ }^{*}$ kawabori.masashi@gmail.com
}

Received 9 April 2014; revised 9 May 2014; accepted 10 June 2014

Copyright (C) 2014 by authors and Scientific Research Publishing Inc. This work is licensed under the Creative Commons Attribution International License (CC BY). http://creativecommons.org/licenses/by/4.0/

\section{(c) (i) Open Access}

\begin{abstract}
Purpose: To achieve good outcomes during aortic surgery with circulatory arrest, a secure and non-bleeding anastomosis must be achieved rapidly to ensure brain protection. We report our initial experiences with a modified Branched Graft Inverting (BGI) technique using an inserter under mild hypothermia. We aimed to reduce the surgical duration and to prevent unnecessary damage to the fragile aorta. Methods: We retrospectively reviewed patients with type A acute aortic dissection (AAD) who underwent distal anastomosis via the modified BGI technique using an inserter between January 2012 and March 2013. Open distal anastomosis was performed under mild hypothermia with right hemisphere perfusion from the right axillary artery. Results: Eight patients were enrolled. There was no mortality. Circulatory arrest time was reproducibly $20.3 \pm 1.9 \mathrm{~min}$, which was sufficient to complete non-bleeding distal anastomoses. The average rectal temperature during circulatory arrest was $26.5^{\circ} \mathrm{C} \pm 1.9^{\circ} \mathrm{C}$. All patients were extubated the day after the operation without any neurological deficit. Conclusion: The modified BGI technique employing an inserter and mild hypothermia offered easy, secure, and reproducible distal anastomosis for ascending aortic replacement for type A acute aortic dissection. Our outcomes were favorable and support further development of this technique.
\end{abstract}

\section{Keywords}

Aortic Dissection, Ascending Replacement, Syringe, Branched Graft Inversion, Mild Hypothermia

\footnotetext{
${ }^{*}$ Corresponding author.
}

How to cite this paper: Kawabori, M., Murakami, T., Ahn, K., Kato, Y., Kotani, M. and Toyama, M. (2014) A Modified Branched Graft Inversion Technique Employing a Syringe Inserter in Ascending Aortic Replacement for Type A Aortic Dissection. World Journal of Cardiovascular Surgery, 4, 95-100. http://dx.doi.org/10.4236/wjcs.2014.47015 


\section{Introduction}

To achieve good outcomes during aortic surgery with circulatory arrest, such as open distal anastomosis for type A acute aortic dissection, a secure and non-bleeding anastomosis must be achieved rapidly to ensure brain protection. Typically, a two-step method is adopted that involves aortic stump reinforcement and graft anastomosis, which can prolong both the surgical duration and circulatory arrest time. The extent of aortic replacement and surgical duration have been reported as significant risk factors for hospital mortality [1]-[5] because patients may be unable to tolerate the surgical stress of deep hypothermia (DHT), long durations of cerebral exclusion, or cardiopulmonary bypass. Additionally, prolonged surgery can increase mortality through coagulopathy, cerebral ischemia, infection, or multiple organ failure. This is supported by Cook et al. [6] who reported that decreasing the systemic temperature to below $22^{\circ} \mathrm{C}$ may be unnecessary and associated with a higher incidence of neurologic injury during DHT. A low invasive quick repair (LIQR) technique was reported by Hata et al. [7] [8] who found that $20 \mathrm{~min}$ of mild hypothermic circulatory arrest (rectal temperature, $28^{\circ} \mathrm{C}$ ) without any cerebral perfusion is safe, and resulted in better clinical outcomes than with cooling to DHT without selective cerebral perfusion. We believe that it is possible to reduce the length of the procedure further by combining aortic stump reinforcement and graft anastomosis in a single step by using our Branched Graft Inverting (BGI) technique which will lead to shorter circulatory arrest times and less marked hypothermia that is closer to normal physiological levels.

The use of an inverted graft that was invaginated into an injection syringe before being atraumatically injected into the aorta, yielded promising results [9]. Therefore, we have developed a modified BGI technique under mild hypothermia that uses an inserter to enable us to set the graft in proper position in fast and secure way and perform reinforcement of the aortic stamp and graft anastomosis to be completed simultaneously. This was expected to result in shorter circulatory arrest times and to allow for modification of the cerebral protection strategy. Furthermore, we anticipated that the inserter would allow the procedure to be performed without damaging the fragile dissected aorta. Our best efforts are necessary to avoid further damage because the dissected aorta may dilate and progress to a dissected aortic aneurysm over time. We also anticipate that the proposed technique should result in less bleeding, because the device is expected to protect against the damaging effects of graft insertion.

\section{Methods}

\subsection{Study Design}

This was a retrospective observational study reporting our initial experiences with a modified BGI technique using an inserter under mild hypothermia. The modified technique employed an inserter designed to allow reinforcement of the aortic stump while simultaneously performing distal graft anastomosis. The primary outcome was to determine feasibility of the technique, and the secondary outcomes were the safety and operative parameters. We retrospectively reviewed the medical records of all the patients before surgery. Additionally, all patients received contrast-enhanced computed tomography (CT) followed by transthoracic echocardiography to detect pericardial effusion, aortic valve regurgitation (AR), and to assess cardiac function. Data were expressed as mean \pm standard deviation for continuous variables and as percentages for categorical variables.

\subsection{Patient Selection}

We enrolled patients who underwent ascending aortic replacement for acute aortic dissection using the modified BGI technique at our institution between April 2012 and April 2013. Patients were excluded if they required aortic arch replacement or had severe atherosclerotic changes in the aortic arch.

\subsection{Surgical Procedure}

\subsubsection{Graft Preparation}

A single branched $26 \mathrm{~mm}$ Triplex graft (Terumo Corp, Tokyo, Japan) was cut $4 \mathrm{~cm}$ proximal and $2 \mathrm{~cm}$ distal to the branch, resulting in a total length of approximately $7 \mathrm{~cm}$. Further, the graft was completely inverted (Figure 1(a)). We created an inserter by severing the tip of a $10 \mathrm{~mL}$ injection syringe (Terumo Corp, Tokyo, Japan) with scissors and then placed the invaginated vascular graft within the inserter (Figure 1(b)). 


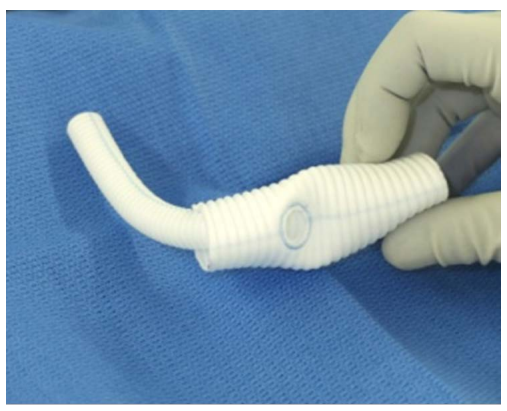

(a)

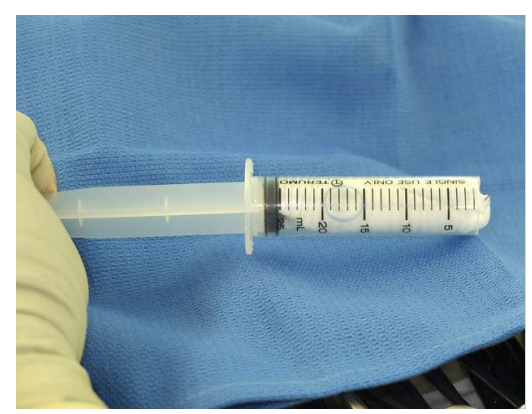

(b)

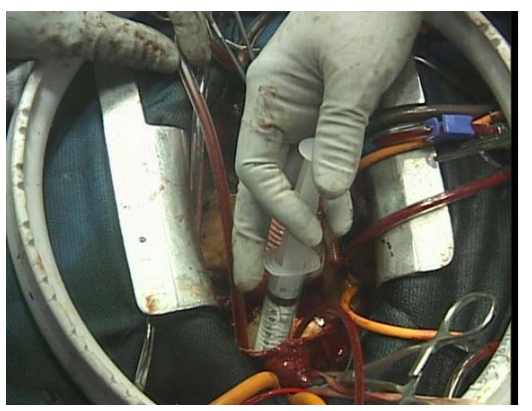

(c)

Figure 1. (a) Preparation of the graft. The branched graft is cut and completely inverted; (b) The inverted graft is invaginated into the severed syringe and prepared for deployment; (c) By withdrawing the barrel with the plunger fixed, the graft is atraumatically placed in the aorta.

\subsubsection{Bypass and Cooling}

The right axillary and femoral arteries were cannulated for perfusion. Cardiopulmonary bypass was initiated with right atrial drainage. Next, cooling was initiated, during which the ascending aorta was cross-clamped, and the proximal stump of the aorta was reinforced and anastomosed with a graft. When the core temperature reached $26^{\circ} \mathrm{C}$, the carotid branches were clamped and circulatory arrest was initiated. Right cerebral hemisphere perfusion was continued from the right brachial artery for selective cerebral perfusion.

\subsubsection{Graft Insertion}

The ascending aorta was transected $1 \mathrm{~cm}$ proximal to the origin of the brachiocephalic artery. Next, we carefully introduced the inserter holding the inverted graft into the aorta. After withdrawing the syringe barrel with the plunger fixed, the inverted graft was placed in the aorta (Figure 1(c)).

\subsubsection{Completing the Procedure}

After insertion the graft was evenly fixed using four felt-pledgeted sutures. Distal anastomosis was performed using 3 - 0 polypropylene running sutures buttressed with a Teflon felt strip outside the aorta (Figure 2). We added extra sutures as necessary to establish non-bleeding anastomosis. After performing anastomosis, the inverted graft was removed proximally. Soft plaques were flushed out by retrograde aortic perfusion from the femoral artery. Antegrade perfusion was performed from the graft branch and the patient was rewarmed. Finally, anastomosis between proximal and distal grafts was completed.

\section{Results}

\subsection{Descriptive Statistics}

We identified 8 patients who met the inclusion and exclusion criteria; their preoperative profiles are shown in Table 1. A retrospective review of their medical records revealed that $75 \%$ were male, and that the mean patient age was $62.1 \pm 6.4$ years (range, 51 - 77 years). The contrast-enhanced CT results revealed that all 8 cases were communicating aortic dissections. Preoperative complications included shock due to cardiac tamponade $(\mathrm{n}=1)$, moderate AR $(n=4)$, cerebral ischemia $(n=2)$, and unconsciousness $(n=1)$.

\subsection{Perioperative Outcomes}

There were no mortalities. Although open distal anastomosis with the BGI technique was performed by 4 different surgeons (including a resident), the circulatory arrest time was short at $20.3 \pm 1.9 \mathrm{~min}$ (range, 18 - $24 \mathrm{~min}$ ) (Table 2). The mean rectal temperature during circulatory arrest was $26.5^{\circ} \mathrm{C} \pm 1.9^{\circ} \mathrm{C}$, and approximately 20 min was needed to ensure non-bleeding anastomosis.

\subsection{Postoperative Outcomes}

Postoperative complications were minimal. There were no neurologic complications related to surgical 


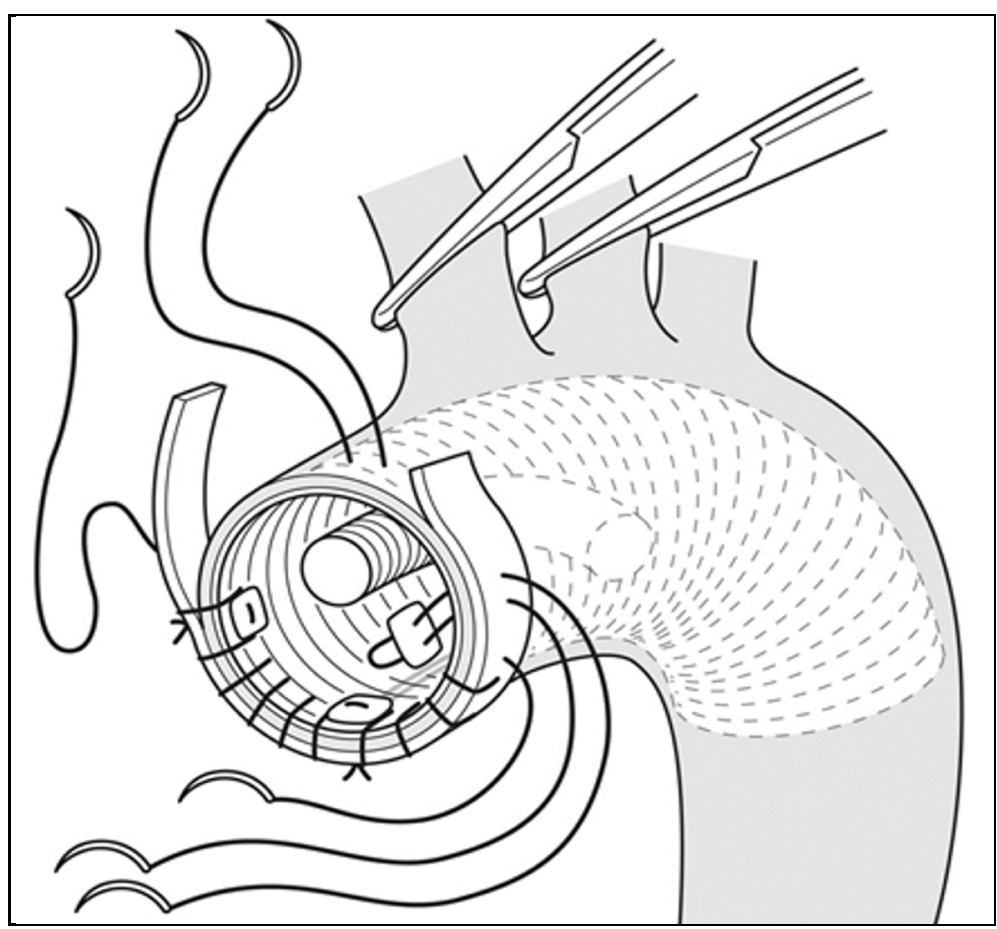

Figure 2. The distal anastomosis performed after insertion of the inverted graft into the aorta.

Table 1. Preoperative patient characteristics and complications.

\begin{tabular}{cc}
\hline Demographics & \\
\hline age (years) & $62.1 \pm 6.4$ \\
Sex (male:female) & $6: 02$ \\
Preoperative complication & \\
Occlusion type & 0 \\
AR & $4(50 \%)$ \\
shock by cardiac tamponade & $1(12.5 \%)$ \\
AMI & 0 \\
Brachiocephalic artery occlusion & 2 \\
coronary dissection & 1 \\
CRF & 0 \\
Cerebral ischemia & $2(25 \%)$ \\
\hline
\end{tabular}

Table 2. CA time by 4 surgeon performing BGI technique.

\begin{tabular}{ccc}
\hline Pt & CA (min) & Operator \\
\hline 1 & 19 & Chief \\
2 & 22 & Surgeon A \\
3 & 20 & Surgeon A \\
4 & 23 & Surgeon B \\
5 & 22 & Surgeon B \\
6 & 18 & Chief \\
7 & 23 & Surgeon A \\
8 & 24 & Surgeon C \\
\hline
\end{tabular}


procedures and no re-operations to control bleeding. All patients were extubated the day after surgery without neurological deficit. One patient developed a stroke 12 days postoperatively.

The mean duration of stay in the intensive care unit was $3.7 \pm 1.9$ days, and the mean duration of hospitalization was 14.0 days. Related procedures included fixation of detached aortic commissures $(n=4)$, brachiocephalic artery reconstruction $(n=2)$, and coronary artery bypass grafting $(n=1)$.

\section{Discussion}

The extent of aortic replacement and surgical duration are significant risk factors for hospital mortality [1]-[5]. This initial report has demonstrated that the modified BGI technique enabled us to reinforce the aortic stump while simultaneously performing distal graft anastomosis. Thus, we were easily able to accomplish secure and reproducible distal anastomosis. As evidenced by the low morbidity and zero mortality, the procedure appears to be safe. Of note, we experienced no difficulties with bleeding, which meant that no patients underwent re-exploration for this complication.

It is possible that our results were favorable because of the target level and duration of hypothermia. Indeed, we had no bleeding or neurological complications as stated. The evidence now suggests that decreasing the systemic temperature to below $22^{\circ} \mathrm{C}$ may be unnecessary and associated with a higher incidence of neurologic injury during DHT [6]. There was little difference between the circulatory arrest times for the distal anastomoses: we found that a circulatory arrest time of approximately 20 min was sufficient to close non-bleeding anastomoses, and that at least 20 min was needed for minimally bleeding anastomoses. This is consistent with the findings of Hata et al. [7] [8] who reported the safety and superiority of circulatory arrest for $20 \mathrm{~min}$ at $28^{\circ} \mathrm{C}$. At our institution, the patients were cooled to a rectal temperature of $26^{\circ} \mathrm{C}$. Furthermore, an additional right hemisphere perfusion was adopted to increase the procedure safety during prolonged circulatory arrest. The clinical outcomes were promising, and revealed no mortality, neurological dysfunction, or complications directly related to the procedure.

Although this modified BGI technique can lessen the impact of surgery and hypothermia related stress, it cannot be used for severely atherosclerotic or "shaggy" aortas because of the risk of embolism. However, it is sometimes difficult to insert an inverted graft into a fragile dissected aorta, and the aortic arch can be damaged if the graft is inserted without care. The use of an injection syringe for graft insertion in non-bleeding anastomoses was successful in this regard. This is because the inverted graft is invaginated into an injection syringe and atraumatically injected into the aorta [9].

Finally, this study does have limitations. Notably, the sample size was small $(n=8)$, the study was not controlled, and we performed no statistical analysis. Consequently, the present data are insufficient to claim definitively that this procedure is effective. However, this study does provide the foundation for further investigation.

\section{Conclusion}

Our technique offers an easy, secure, and reproducible method for performing non-bleeding distal anastomosis. We could secure non-bleeding anastomosis within $20 \mathrm{~min}$, ensuring safe circulatory arrest under mild hypothermia. Shorter circulatory arrest times and mild hypothermia probably contributed to the low rate of postoperative complications. Therefore, we consider that under mild hypothermia, this technique was useful for ascending aortic replacement in type A acute aortic dissection. However, further studies with larger cohorts that compare outcomes against the standard procedure are necessary to confirm these initial findings.

\section{Conflict of Interest Disclosure Statement}

The author declares no conflicts of interest.

\section{References}

[1] Kawahito, K, Adachi, H, Yamaguchi, A. and Ino, T. (2001) Preoperative Risk Factors for Hospital Mortality in Acute Type A Aortic Dissection. The Annals of Thoracic Surgery, 71, 1239-1243. http://dx.doi.org/10.1016/S0003-4975(00)02654-0

[2] Ehrlich, M.P., Ergin, M.A., McCullough, J.N., Lansman, S.L., Galla, J.D., Griepp, R.B., et al. (2000) Result of Immediate Surgical Treatment of All Acute Type A Dissections. Circulation, 102, 248-252. 


\section{http://dx.doi.org/10.1161/01.CIR.102.suppl_3.III-248}

[3] Kamiya, H., Hagl, C., Kropivnitskaya, I., Weiderwann, J., Kallenbach, K., Haverich, A., et al. (2007) Quick Proximal Arch Replacement with Moderate Hypothermic Circulatory Arrest. The Annals of Thoracic Surgery, 83, 1055-1058. http://dx.doi.org/10.1016/j.athoracsur.2006.09.085

[4] Hata, M., Suzuki, M., Sezai, A., Niino, T., Yoshitake, I., Minami, K., et al. (2008) Less Invasive Quick Replacement for Octogenarians with Type A Acute Aortic Dissection. The Journal of Thoracic and Cardiovascular Surgery, 136, 489-493. http://dx.doi.org/10.1016/j.jtcvs.2008.01.007

[5] Sabik, J.F., Lytle, B.W., Blackstone, E.H., McCarthy, P.M., Loop, F.D. and Cosgrove, D.M. (2000) Long-Term Effectiveness of Operations for Ascending Aortic Dissections. The Journal of Thoracic and Cardiovascular Surgery, 119, 946-962. http://dx.doi.org/10.1016/S0022-5223(00)70090-0

[6] Cook, R.C., Gao, M., Macnab, A.J., Fedoruk, L.M., Day, N. and Janusz, M.T. (2006) Aortic Arch Reconstruction: Safety of Moderate Hypothermia and Antegrade Cerebral Perfusion during Systemic Circulatory Arrest. The Journal of Cardiovascular Surgery, 21, 158-164.

[7] Hata, M., Sezai, A., Yoshitake, I., Wakui, S., Takasaka, A., Minami, K. and Shiono, M. (2010) Clinical Trends in Optimal Treatment Strategy for Type A Acute Aortic Dissection. Annals of Thoracic and Cardiovascular Surgery, 16, 228-235.

[8] Hata, M., Suzuki, M., Sezai, A., Niino, T., Yoshitake, I., Shimura, K. and Minami, K. (2009) Outcome of Less Invasive Proximal Arch Replacement with Moderate Hypothermic Circulatory Arrest Followed by Aggressive Rapid ReWarming in Emergency Surgery for Type A Acute Aortic Dissection. Circulation Journal, 73, 69-72. http://dx.doi.org/10.1253/circj.CJ-08-0499

[9] Ishikawa, N., Omoto, T., Miyauchi, T., Oi, M., Fukuzumi, M. and Tedoriya, T. (2012) Simple and Safe Graft Insertion in the Elephant Trunk Technique. Asian Cardiovascular and Thoracic Annals, 20, 356-357.

http://dx.doi.org/10.1177/0218492311436121 
Scientific Research Publishing (SCIRP) is one of the largest Open Access journal publishers. It is currently publishing more than 200 open access, online, peer-reviewed journals covering a wide range of academic disciplines. SCIRP serves the worldwide academic communities and contributes to the progress and application of science with its publication.

Other selected journals from SCIRP are listed as below. Submit your manuscript to us via either submit@scirp.org or Online Submission Portal.
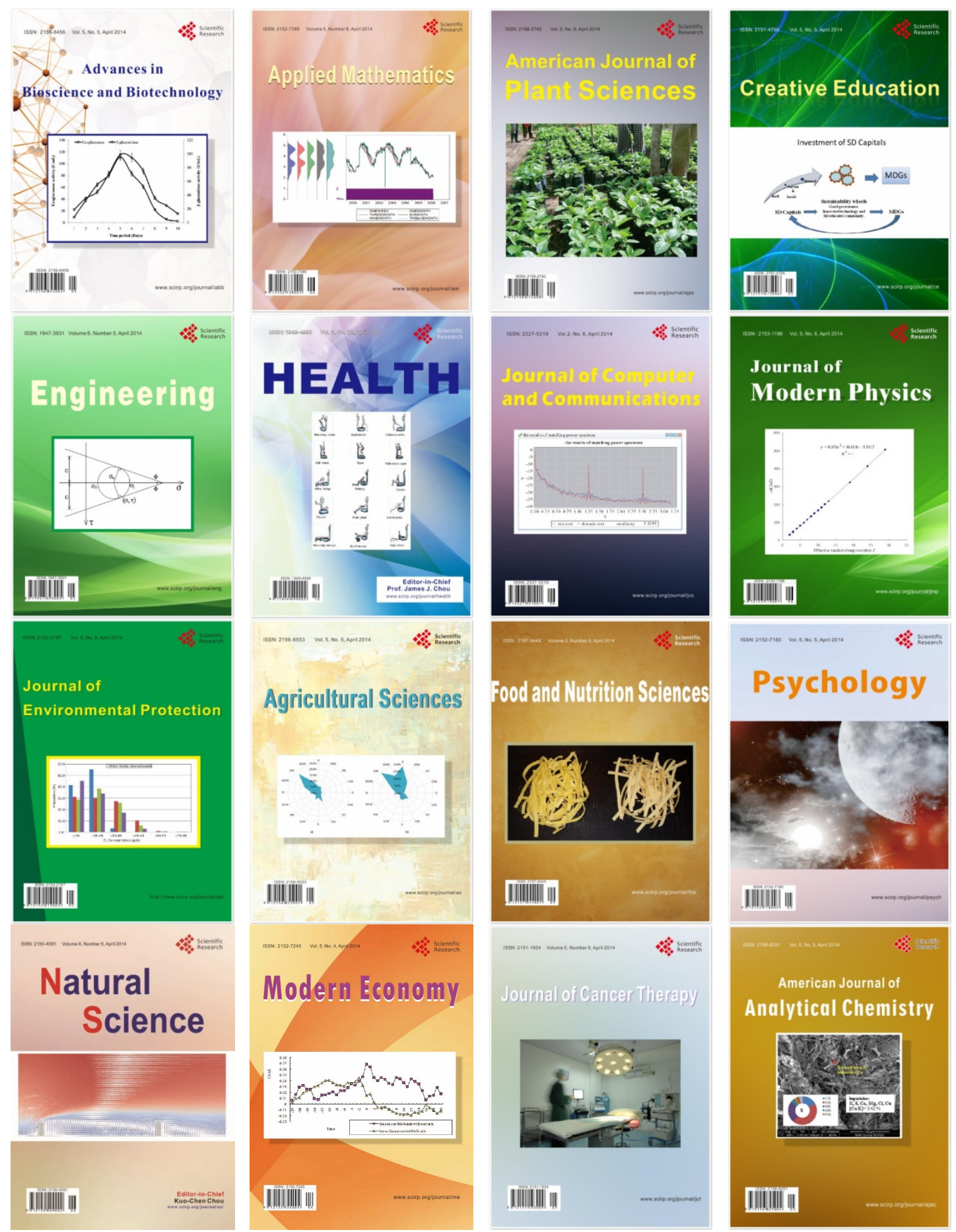